Pure and Applied Mathematics Quarterly

Volume 3, Number 3

(Special Issue: In honor of

Leon Simon, Part 2 of 2$)$

$737-772,2007$

\title{
Geometry, Topology, and Gravitation Synthesized by Cosmic Strings*
}

\author{
Yisong Yang
}

\begin{abstract}
In the context of static cosmic strings, the Einstein equations coupled to the Abelian Higgs model and the gauged $\sigma$-model reduce into a single equation, which directly relates the Gauss curvature of a Riemann surface hosting nontrivial geometry and gravitation to the energy density of the coupled matter-gauge sector. This equation gives rise to an equivalence relation between the topology of the Riemann surface and the topology of the complex line bundle which hosts and is determined by the physical interactions of the matter-gauge sector. The existence of solutions realizing a prescribed distribution of cosmic strings resembles the classical prescribed Gauss curvature problem.
\end{abstract}

\section{Geometry, Physics, and Governing Equations}

Geometry, Physics, and the Einstein Equations. The basic principle that led Einstein to write down his fundamental equations for gravitation is that the geometry of spacetime which governs the gravitation in it is determined by the matter it contains. These equations may be viewed as the equations of motion of

Received December 9, 2005.

*This survey article is written based on a talk given at the conference Partial Differential Equations and Calculus of Variations in honor of Professors Robert Gulliver, Robert Hardt, and Leon Simon on the occasion of their 60th birthdays, Zhejiang University, Hangzhou, China, June 23-28, 2005. 
spacetime and are of the form

$$
R_{\mu \nu}-\frac{1}{2} g_{\mu \nu} R=-8 \pi G T_{\mu \nu}
$$

Here $g_{\mu \nu}$ is the metric tensor of the spacetime with signature $(+---), R_{\mu \nu}$ is the Ricci tensor, $R$ is the scalar curvature, $R_{\mu \nu}-\frac{1}{2} g_{\mu \nu} R=G_{\mu \nu}$ is the Einstein tensor, $T_{\mu \nu}$ is the energy-momentum tensor of the matter (and gauge field) sector, and $G>0$ is the Newton universal gravitational constant. Note that $T_{\mu \nu}$ in turn is determined by the equations of motion defined over the gravitational metric background of the spacetime. In this way, gravitation and matter are closely coupled together.

Spacetime Geometry and Topology. It is also well known that geometry and topology of spacetime are often tightly related to each other. A classical example is the Chern-Gauss-Bonnet theorem which says that the integral of the Pfaffian $P f(\Omega)$, a $2 n$-form constructed from the $s o(2 n)$-valued curvature 2 -form of the Levi-Civita connection of a compact Riemannian manifold $M$ of dimension $2 n$, gives rise to the Euler characteristic of $M$,

$$
\int_{M} \operatorname{Pf}(\Omega)=(2 \pi)^{n} \chi(M) .
$$

Matter Sector and Bundle Topology. On the other hand, all the other fundamental physical forces than gravity are housed within a sufficiently large principal bundle over the spacetime manifold. An elegant example is the Yang-Mills instantons housed in an $S U(2)$-bundle over the standard 4-sphere $\left(S^{4}, g\right)$ ( $g$ is the metric of $\left.S^{4}\right)$. The energy (action) functional governing an $s u(2)$-valued gauge connection $A$ is defined by

$$
E(A)=-\int_{S^{4}} \operatorname{Tr}\left(F_{A} \wedge *_{g} F_{A}\right),
$$

where $*_{g}$ is the Hodge dual induced from $g$ and $F_{A}=\mathrm{d} A+A \wedge A$ is the curvature. One is interested in the global minimizers of (1.3) among the topological class that the associated Chern or Pontryagin class of the curvature is a prescribed integer,

$$
c_{2}\left(F_{A}\right)=p_{1}\left(F_{A}\right)=-\frac{1}{8 \pi^{2}} \int_{S^{4}} \operatorname{Tr}\left(F_{A} \wedge F_{A}\right)= \pm N,
$$

where $N \in \mathbb{N}$. It is well known that for (1.3) subject to (1.4) there holds the topological energy lower bound $E \geq 8 \pi^{2} N$. This lower bound is saturated if and 
only if the connection $A$ solves the self-dual or anti-self-dual equation

$$
F_{A}= \pm *_{g} F_{A},
$$

which may be viewed as a first integral of the original second-order equations of motion of the energy (1.3), and the curvature $F_{A}$ belongs to the class designated by (1.4). See $[1,54,61]$ and references therein.

Differential Equations. Traditionally, the study on the gravitational equations (1.1) and on the equations of motion of other fundamental forces such as (1.5) are conducted following parallel paths: when one studies (1.1), one tries to understand the geometry/gravitation of the spacetime defined by its metric $g$ which is a solution of (1.1) subject to a prescribed energy-momentum tensor $T_{\mu \nu}$ of the matter or particle-physics sector; when one studies (1.5), one tries to understand the solution structure of (1.5) assuming that the underlying spacetime metric $g$ is given as a background metric. Due to the difficulty one encounters, there have been very few attempts to understand truly coupled gravitational and physical systems such as a system comprised of (1.1) and (1.5) (say), which is of obvious importance for cosmology. In [69], the existence of regular static solutions to the Einstein and $S U(2)$ Yang-Mills equations over a $(3+1)$-dimensional Lorentzian spacetime is established under a radially symmetric field configuration ansatz.

Cosmic Strings and the Einstein Equations. Here we are interested in another class of static solutions to the coupled Einstein and matter-gauge equations called cosmic strings $[27,30,43,44,50,51,72,73,77]$ which incorporate the simplest topological invariants determined geometrically via formulas of the form (1.2) and (1.4). According to physicists, cosmic strings are hypothetical massive objects that may have formed shortly after the big bang and can be thought of as tubular samples of the universe from about 10-35 second after the beginning of time. They are predicted to be infinitesimally small in cross section but enormously long, perhaps forming loops that could encircle an entire galaxy. They would also be extremely massive - a one-meter length might weigh 1.6 times as much as the earth and, consequently, give rise to intense and very strange gravitational fields. They provide one possible solution to the problem of dark matter in the universe and have been posited as the seeds around which galaxies formed [75]. Mathematically, the simplest cosmic strings are straight and infinitely long. In this situation, although the spacetime is 4-dimensional, the nontrivial geometry of cosmic strings is actually 2-dimensional and the spacetime is assumed to be 
uniform along the time axis and a spatial axis and gravitation is induced from a 2-surface, $S$. As a consequence, the Einstein tensor on the left-hand side of (1.1) has only two nonvanishing components which are related to the unknown metric $g$ of $S$ through its Gauss curvature $K_{g}$ and (1.1) becomes a scalar equation

$$
K_{g}=8 \pi G H
$$

where $H$ is the Hamiltonian (energy) density of the matter-gauge fields sector governed by the static equations of motion over $(S, g)$ of the form

$$
\delta \int_{S} H \mathrm{~d} V_{g}=0
$$

where $\delta$ denotes the Fréchet derivative. Note that we will also use $\delta$ to denote the standard Euclidean metric $\left(\delta_{j k}\right)$ or the deficit angle of conical infinity in other parts of this article. In other words, the coupled Einstein and matter-gauge equations are reduced to the system of equations (1.6) and (1.7). Cosmic strings are generated by solutions with localized concentration of the energy density $H$, which through (1.6), immediately give rise to the localized concentration of the Gauss curvature $K_{g}$ as expected by cosmologists. Such locally concentrated solutions, often called solitons in particle physics, have interesting topological origins in the bundle that houses the concerned matter and gauge fields. For this reason, they are also called topological defects [30, 43, 44, 72, 73, 77].

Prescribed Gauss Curvature Equation. The equation (1.6) may be compared with the classical Prescribed Gauss Curvature Problem in geometric analysis $[7,9,10,12,22,23,25,26,33,39,40,41,42,47,52,55]$,

$$
K_{g}(x)=F(x),
$$

where $F(x)$ is a given scalar function over the underlying surface $\left(S, g_{0}\right)$ and one asks the question whether one can get a metric $g$ among the conformal class of $g_{0}$ so that the Gauss curvature $K_{g}$ of $(S, g)$ coincides everywhere on $S$ with $F$.

Comparison and Discussion. In view of (1.8), the system of equations (1.6) and (1.7) is a new prescribed curvature problem. In this context, the data to be prescribed will no longer be the curvature but are the cosmic strings with their respective local string strength. More specifically, we are interested in the question whether there exists a solution to (1.6) and (1.7) realizing cosmic strings 
prescribed at the points $p_{1}, \cdots, p_{\ell} \in S$ with their respective string local winding numbers $n_{1}, \cdots, n_{\ell}$. We shall see that, in view of these solutions, geometry/gravitation and topologies both at the spacetime level and the bundle level are closely related as well as a consequence of our cosmic string contents. We choose two well known examples to illustrate such a picture. The first example is the classical Abelian Higgs model $[38,70]$ also known as the Ginzburg-Landau theory for superconductivity $[20,28,32,48,78,79]$, which is a simplest YangMills theory model, and the cosmic strings $[80,81,82]$ are simply gravitating superconducting vortices [77]. The second example is the gauged $\sigma$-model [66, 67] which allows the coexistence of magnetic vortices and antivortices and may be used to obtain cosmic strings with opposite string charges [84, 85, 88]. The equation (1.6) relates the topology of the underlying surface $S$ via the GaussBonnet formula, the simplest form of the formula (1.2), to the topology of a complex line bundle over $S$, which hosts the matter-gauge interaction, via the minimum energy of the matter-gauge system, whose value is given by the first Chern class (say). In the next section, we review the static solutions of the 2dimensional Abelian Higgs model and of the gauged $\sigma$-model when gravity is not present. In Section 3, we consider cosmic strings which are in fact vortices which give rise to gravitation through introducing nontrivial geometry and topology. After stating the main existence theorem $[80,81]$ concerning cosmic strings in the Abelian Higgs model within a noncompact setting, we illustrate the results using the explicit construction in [27] for solutions of the (bare) $\sigma$-model. We then state our existence theorem for cosmic string solutions of the Abelian Higgs model within a compact setting [81, 82]. We also present a discussion comparing our structure with that in more familiar problems in three dimensions, the Positive Energy Theorem [59, 62, 63, 64, 65, 76] and the Penrose Conjecture [60] in general relativity. In Section 4, we consider the coexistence problem of cosmic strings and antistrings $[84,85,88]$ in the gauged $\sigma$-model $[66,67]$. We present some details about the PDE structure of the problem. In particular, we show that the existence proof in the compact setting case relies on our understanding of an interesting nonlinear elliptic equation arising in condensed-matter physics, namely, the self-dual Chern-Simons equation [29, 34, 37], which may be solved by minimizing an energy functional subject to an inequality constraint $[21,87]$. Section 5 contains some concluding comments. These results can be extended to the case when electromagnetism is governed nonlinearly by the Born-Infeld 
dynamics $[14,15,45,46,49,86]$. However, there are still considerable difficulties when we consider cosmic strings generated by non-Abelian gauge field theory models such as those in the electroweak theory $[2,3,4,5,83,87]$.

\section{Solitons in Absence of Gravity}

In this section, we review some results concerning the soliton solutions to the matter-gauge equations defined by (1.7) when the background two-surface $(S, g)$ is a given Riemann surface (compact or Euclidean). Since the metric $g$ is known, we only use $*$ (instead of $*_{g}$ ) here to denote the Hodge dual. For simplicity, we shall only consider several Abelian cases. These 2D solitons are often called vortices.

Abelian Higgs Vortices. Let $\xi$ be a complex line bundle over the Riemann surface $S$. Use $u$ to denote a section $\xi \rightarrow S$. If $A$ is a real-valued (Abelian) connection 1-form, then $D_{A} u=\mathrm{d} u-\mathrm{i} A u$ defines an induced connection and $F_{A}=\mathrm{d} A$ is the curvature 2-form. The Hamiltonian density $H$ of the Abelian Higgs theory is written as

$$
H(u, A)=\frac{1}{2} *\left(F_{A} \wedge * F_{A}\right)+\frac{1}{2} *\left(D_{A} u \wedge * \overline{D_{A} u}\right)+\frac{1}{8}\left(1-|u|^{2}\right)^{2} .
$$

We are to find the global minimizers of the energy

$$
E(u, A)=\int_{S} H(u, A) \mathrm{d} V
$$

subject to the topological constraint

$$
c_{1}(\xi)=\frac{1}{2 \pi} \int_{S} F_{A}= \pm N,
$$

where $c_{1}(\xi)$ is the first Chern class of the line bundle and $N$ is a given positive integer. In view of the procedure of Bogomol'nyi [13], it can be shown that there holds the topological energy lower bound,

$$
E(u, A)=\int_{S} H(u, A) \mathrm{d} V \geq \pi\left|c_{1}(\xi)\right|=\pi N
$$

and the equality is saturated if and only if $(u, A)$ satisfies the self-dual or antiself-dual equations [13, 16, 38, 56, 74]

$$
\begin{aligned}
D_{A} u \pm \mathrm{i} * D_{A} u & =0, \\
F_{A} & = \pm \frac{1}{2} *\left(1-|u|^{2}\right),
\end{aligned}
$$


over $S$. Besides, the integer $N$ is nothing but $N$ zeros of the section $u$ which may in fact be arbitrarily prescribed over $S$. It is seen from the second equation in (2.5) that the zeros of $u$ give the absolute maxima $1 / 2$ of the "magnetic" or "vorticity" field $* F_{A}$ and of the potential density $\frac{1}{8}\left(1-|u|^{2}\right)^{2}$, which give rise to the locally peaked values of the energy density $H(u, A)(x)$ as expected. Hence, we are led to the following problem.

Prescribed Vortex Problem. Given the points $p_{1}, \cdots, p_{\ell} \in S$ and integers $n_{1}, \cdots, n_{\ell} \in$ $\mathbf{N}$, obtain a solution $(u, A)$ of the system $(2.5)$ so that $u\left(p_{s}\right)=0$ and the zero $p_{s}$ of $u$ has an algebraic multiplicity $n_{s}, s=1, \cdots, \ell$, and $n_{1}+\cdots+n_{\ell}=N$ (in the case that $S$ is the Euclidean space $\mathbb{R}^{2}$, we need to require that the solution be of finite energy, $E(u, A)<\infty)$. In the context of the theory of superconductivity, $u$ is an order parameter and $u \neq 0$ indicates the onset of superconducting phase and $u=0$ characterizes the destruction of superconducting phase or the onset of normal phase. Hence, the zeros of $u$ are called the "defects" in a superconductor through which maximum penetration of magnetic field into the superconductor takes place.

The above problem has already been completely solved: when $S=\mathbb{R}^{2}$, the problem always has a unique solution $[38,70]$; when $S$ is a compact surface with total surface area $|S|$, the problem has a solution (which is unique if exists) if and only if $[16,31,56,74]$

$$
N<\frac{|S|}{4 \pi}
$$

The condition (2.6) may be interpreted as a "living space" constraint and may be easily seen from integrating the second equation in (2.5): Abelian Higgs vortices need space to stay together and surfaces with larger surface areas allow more vortices. In particular, when the surface area becomes infinite such as in the case of $S=\mathbb{R}^{2}$, any finite number of vortices for a solution is allowed.

Vortices and Antivortices in the Gauged $\sigma$-Model. The original gauged $\sigma$-model introduced by Schroers [66, 67], which extends the classical sigma model [11, 61] (harmonic maps), includes a map from $\mathbb{R}^{2}$ to $S^{2}$ and an Abelian gauge field $A$. In [68], we use the stereographic projection to complexify the target space $S^{2}$ and we naturally arrive at an Abelian gauge field theory defined by the Hamiltonian 
density

$$
H(u, A)=\frac{1}{2} *\left(F_{A} \wedge * F_{A}\right)+2 *\left(\frac{D_{A} u \wedge * \overline{D_{A} u}}{\left(1+|u|^{2}\right)^{2}}\right)+\frac{1}{2}\left(\frac{1-|u|^{2}}{1+|u|^{2}}\right)^{2},
$$

formulated as before over a complex line bundle $\xi \rightarrow S$. In this situation, we are interested in a global minimizer $(u, A)$ of the energy subject to a suitable topological constraint so that, algebraically, $u$ has $N$ zeros and $P$ poles at the prescribed locations. It has been observed [68] that the first Chern class $c_{1}(\xi)$ alone is not sufficient to characterize such a solution and another topological invariant, the Thom class $\tau\left(\xi^{*}\right)$ of the dual bundle $\xi^{*} \rightarrow S$, comes into play. In fact, these two topological invariants are related to the integers $N$ and $P$ through the formulas

$$
\begin{aligned}
c_{1}(\xi) & =\frac{1}{2 \pi} \int_{S} F_{A}= \pm(N-P), \\
\tau\left(\xi^{*}\right) & =\frac{1}{4 \pi} \int_{S} J_{A}(u)= \pm P
\end{aligned}
$$

where the new "vorticity field" $J_{A}(u)$ is defined by

$$
J_{A}(u)=-\frac{2|u|^{2}}{1+|u|^{2}} F_{A}+\mathrm{i}\left(\frac{D_{A} u \wedge \overline{D_{A} u}-\left(* D_{A} u\right) \wedge \overline{\left(* D_{A} u\right)}}{\left(1+|u|^{2}\right)^{2}}\right) .
$$

Similar to the Abelian Higgs case, the topological energy lower bound takes the form

$$
E(u, A)=\int_{S} H(u, A) \mathrm{d} V \geq 2 \pi\left|c_{1}(\xi)+2 \tau\left(\xi^{*}\right)\right|=2 \pi(N+P),
$$

with the equality attained if and only if the pair $(u, A)$ satisfies the self-dual or anti-self-dual equations [67, 68, 84, 85]

$$
\begin{aligned}
D_{A} u \pm \mathrm{i} * D_{A} u & =0, \\
F_{A} & = \pm * \frac{1-|u|^{2}}{1+|u|^{2}} .
\end{aligned}
$$

For definiteness, assume the plus sign (the self-dual case) in these equations. It is seen that the magnetic field $* F_{A}$ achieves its global maximum value 1 at a zero of $u$ and its global minimum value -1 at a pole of $u$. Following standard convention to view $* F$ as a vorticity field, then we see that the zeros and poles of $u$ can be interpreted as the centers of vortices and antivortices. As in the Abelian Higgs theory case, the energy density $H(u, A)(x)$ has peaks at the zeros and poles of $u$. 
Prescribed Vortex and Antivortex Problem. Given the points $q_{1}, \cdots, q_{N} \in S$ and $p_{1}, \cdots, p_{P} \in S$, obtain a solution $(u, A)$ of the system $(2.12)$ so that $q_{1}, \cdots, q_{N} \in$ $S$ and $p_{1}, \cdots, p_{P} \in S$ are the zeros and poles of the section $u$ (in the case that $S$ is the Euclidean space $\mathbb{R}^{2}$, we require again the finite-energy condition), respectively.

This problem is also completely solved: when $S=\mathbb{R}^{2}$, for any prescribed data the system has a unique solution $[84,85]$ so that $|u|=1$ at the infinity of $\mathbb{R}^{2}$; when $S$ is a compact surface with the total surface area $|S|$, there is a solution realizing $N$ prescribed zeros and $P$ prescribed poles at arbitrary locations if and only if $N$ and $P$ satisfies the bound [68]

$$
|N-P|<\frac{|S|}{2 \pi}
$$

The condition (2.13) may also be viewed as a similar "living space" constraint: vortices and antivortices do not mind to stay together congestively in a small surface so long as their "gender" discrepancy is not too big and surfaces with larger surface areas allow bigger "gender" discrepancies. In particular, when the surface area becomes infinite such as in the case of $S=\mathbb{R}^{2}$, any finite numbers of vortices and antivortices for a solution are allowed.

As energy densities, the Hamiltonians defined in both (2.1) and (2.7) are positive definite, $H(u, A) \geq 0$, and $H(u, A) \equiv 0$ if and only if the complex line bundle $\xi$ is trivial and $(u, A)$ is gauge-equivalent to the trivial pair, $u=1, A=0$.

\section{Cosmic Strings, Lower Bounds, Etc.}

Positive Gauss Curvature and Topological Lower Bounds. Based on the discussion in the previous section on the Abelian Higgs and gauged $\sigma$-models, we see that the two immediate consequences of the equation (1.6) are

(i) The Gauss curvature $K_{g}$ of $(S, g)$ is always nonnegative,

$$
K_{g} \geq 0 \text {. }
$$

(ii) The integral of the Gauss curvature over $S$, or the total curvature, is bounded from below by a topological invariant, $T$, of the form

$$
\int_{S} K_{g}(x) \mathrm{d} V_{g} \geq C|T|
$$


where $T$ is the first Chern class of the bundle for the Abelian Higgs model, so that $C=8 \pi^{2} G$, and the sum of the first Chern class of the bundle and twice the Thom class of the dual bundle for the gauged $\sigma$-model, so that $C=16 \pi^{2} G$, and the lower bound (3.2) is attained if and only if (2.5) or (2.12) is satisfied.

Cosmic Strings in the Abelian Higgs Model. In the Abelian Higgs model case, we arrive at the coupled system $[27,50,51]$

$$
\begin{aligned}
K_{g} & =8 \pi G H(u, A), \quad(H \text { is as defined in }(2.1)) \\
D_{A} u \pm \mathrm{i} *_{g} D_{A} u & =0, \\
F_{A} & = \pm \frac{1}{2} *_{g}\left(1-|u|^{2}\right) .
\end{aligned}
$$

Thus we are led to use the energy peaks generated by vortices in the last section to produce curvature peaks through the reduced Einstein equation, which is the first equation in (3.3). In other words, we may study the vortex solutions of the Abelian Higgs theory governed by the coupled system (3.3). The solutions are cosmic strings solutions which are also sometimes called gravitating vortices.

Assume that that the 2 -surface $(S, g)$ is not very bizarre. For example, we assume that it is noncompact but complete (without boundary). Using the property (3.1) and the well-known Cohn-Vossen theorem (cf. [24]), we can conclude that $(S, g)$ is either flat or diffeomorphic to $\mathbb{R}^{2}$. Since we are to generate a nontrivial gravitational sector, we see that $S$ must be diffeomorphic to $\mathbb{R}^{2}$. This structure implies that all the components of the unknown gravitational metric may be absorbed into a conformal exponent. Note that we will also obtain solutions which give rise to noncomplete metrics.

Existence of Cosmic Strings in Noncompact Setting. Our results [80, 81] concerning the existence of cosmic string solutions of the system (3.3) can be stated as follows.

Theorem 3.1.. For any prescribed points $p_{1}, \cdots, p_{N} \in S$ (which is necessarily diffeomorphic to $\mathbb{R}^{2}$ ), the system (3.3) has a finite-energy solution $(g, u, A)$ so that $u$ vanishes exactly at $p_{1}, \cdots, p_{N},|u|=1$ at the infinity of $S$, and $g$ is a complete metric for $(S, g)$ if and only if the total string number $N$ and the Newton constant $G$ satisfy

$$
N \leq \frac{1}{4 \pi G}
$$


The surface $S$ has a conical singularity at infinity with a deficit angle $\delta=8 \pi^{2} N G$. In this case the magnetic flux is quantized according to $\Phi=\int_{S} * F_{A} d V_{g}=2 \pi N$ and the total curvature and matter-gauge energy assume their minimum quantized values

$$
\int_{S} K_{g} d V_{g}=8 \pi^{2} G N, \quad \int_{S} H(u, A) d V_{g}=\pi N
$$

If $N=0$, the pair $(u, A)$ is gauge-equivalent to the trivial pair $u=1, A=0$ and $(S, g)$ is globally conformal to the standard Euclidean plane $\left(\mathbb{R}^{2}, \delta\right)$.

We shall not get into a detailed discussion of the technical structure of the above results. Instead, we present as an explicit illustrative example the construction of Comtet and Gibbons [27] of a class of cosmic string solutions generated from the $\sigma$-model of Belavin and Polyakov $[11,61]$ governing the spin vector orientation of a $2 \mathrm{D}$ ferromagnet. In mathematics literature, these solutions are also known as the simplest harmonic maps from $S^{2}$ to itself. Their solutions below share many common features with our solutions and serve as a prelude to our study of the problem of coexistence of cosmic strings and antistrings in the gauged $\sigma$-model.

Explicit Construction of Cosmic String Solutions in the $\sigma$-Model. Let $\phi=$ $\left(\phi_{1}, \phi_{2}, \phi_{3}\right)$ be a map from the spacetime. As before, we replace $\phi$ by its stereographic projection in terms of a complex scalar field $u=u_{1}+\mathrm{i} u_{2}$ where $u_{1}=$ $\phi_{1} /\left(1+\phi_{3}\right)$ and $u_{2}=\phi_{2} /\left(1+\phi_{3}\right)$ and assume $(S, g)=\left(\mathbb{R}^{2}, \mathrm{e}^{\eta} \delta\right)$. Then the static Hamiltonian takes the form

$$
\begin{aligned}
H(\phi) & =\frac{2 \mathrm{e}^{-\eta}}{\left(1+|u|^{2}\right)^{2}}\left(\left|\partial_{1} u\right|^{2}+\left|\partial_{2} u\right|^{2}\right) \\
& =\frac{2 \mathrm{e}^{-\eta}}{\left(1+|u|^{2}\right)^{2}}\left|\partial_{1} u \pm \mathrm{i} \partial_{2} u\right|^{2} \pm \mathrm{i} \frac{2 \mathrm{e}^{-\eta}}{\left(1+|u|^{2}\right)^{2}}\left(\partial_{1} u \partial_{2} \bar{u}-\partial_{1} \bar{u} \partial_{2} u\right) .
\end{aligned}
$$

Using (3.6), we find as before the topological lower energy bound

$$
\begin{aligned}
E(\phi) & =\int_{S} H(\phi) \mathrm{d} \Omega_{g}=\int_{\mathbb{R}^{2}} H(\phi) \mathrm{e}^{\eta} \mathrm{d} x \\
& \geq \pm 2 \mathrm{i} \int_{\mathbb{R}^{2}} \frac{\partial_{1} u \partial_{2} \bar{u}-\partial_{1} \bar{u} \partial_{2} u}{\left(1+|u|^{2}\right)^{2}} \mathrm{~d} x= \pm \int_{\mathbb{R}^{2}} \phi \cdot\left(\partial_{1} \phi \wedge \partial_{2} \phi\right) \mathrm{d} x \\
& =4 \pi|\operatorname{deg}(\phi)|,
\end{aligned}
$$

with equality if and only if $u$ satisfies the Cauchy-Riemann equations

$$
\partial_{1} u \pm \mathrm{i} \partial_{2} u=0 .
$$


It is interesting to note that this equation may be viewed as a limiting case of the first equation in (2.5) or the second equation in (3.3) when $g$ is trivial and the gauge field $A$ vanishes. In this sense, solutions of (2.5) or (3.3) generalize harmonic maps.

In view of (3.8), the energy density $H(\phi)$ becomes a total divergence,

$$
\begin{aligned}
H(\phi) & = \pm J_{12}= \pm\left(\partial_{1} J_{2}-\partial_{2} J_{1}\right), \\
J_{k} & =\frac{\mathrm{i}}{1+|u|^{2}}\left(u \partial_{k} \bar{u}-\bar{u} \partial_{k} u\right), \quad k=1,2 .
\end{aligned}
$$

For definiteness, we consider the case with the plus sign. Hence, from (3.8), we see that $u$ is a meromorphic function in $z=x^{1}+\mathrm{i} x^{2}$, which may be chosen to be

$$
u(z)=c\left(\prod_{s=1}^{N}\left(z-q_{s}\right)\right)\left(\prod_{s=1}^{P}\left(z-p_{s}\right)^{-1}\right),
$$

where $c \neq 0$ is a complex parameter and $q$ 's and $p$ 's are the zeros and poles of $u$ in the complex plane. Consequently, we can use the divergence theorem to arrive at

$$
\begin{aligned}
E & =4 \pi \operatorname{deg}(\phi) \\
& =\int_{\mathbb{R}^{2}} J_{12} \mathrm{~d} x \\
& =\lim _{r \rightarrow \infty} \oint_{|z|=r} J_{k} \mathrm{~d} x^{k}-\lim _{r \rightarrow 0} \sum_{s=1}^{P} \oint_{\left|z-p_{s}\right|=r} J_{k} \mathrm{~d} x^{k},
\end{aligned}
$$

where the line integrals are all taken counterclockwise. Using (3.10), we easily see that, near infinity, $J_{k}$ has the asymptotic estimate

$$
J_{k}=\mathrm{O}\left(|z|^{-\alpha}\right), \quad k=1,2, \quad \alpha=1+2 \max \{0, P-N\} .
$$

Hence, if the total number of poles is larger than the total number of zeros in (3.10), namely,

$$
P>N
$$

then the first term on the right-hand side of (3.11) vanishes in view of (3.12). In fact, the condition (3.13) is exactly the one imposed by Belavin and Polyakov [11] in absence of gravity. 
In order to find the rest of the terms on the right-hand side of (3.11), we note that $J_{k}$ has the estimate

$$
J_{k}=-\epsilon_{j k} \frac{2|u|^{2}}{1+|u|^{2}}\left(\frac{y^{j}}{\left|z-p_{s}\right|^{2}}+\mathrm{O}(1)\right), \quad k=1,2
$$

near the points $p_{s}$, where $y^{1}=\operatorname{Re}\left\{z-p_{s}\right\}$ and $y^{2}=\operatorname{Im}\left\{z-p_{s}\right\}, s=1,2, \cdots, P$. By virtue of (3.14) and the divergence theorem, we obtain

$$
\lim _{r \rightarrow 0} \oint_{\left|z-p_{s}\right|=r} J_{k} \mathrm{~d} x^{k}=\lim _{r \rightarrow 0}\left(\frac{2}{r^{2}} \oint_{\left|z-p_{s}\right|=r} y^{2} \mathrm{~d} y^{1}-y^{1} \mathrm{~d} y^{2}\right)=-4 \pi .
$$

Inserting (3.15) into (3.11), we see that $E=4 \pi P$ and $\operatorname{deg}(\phi)=P$ as expected.

We are now left with the single equation (1.6). With $|u|^{2}=\mathrm{e}^{v}$, we have $\left|\partial_{1} u\right|^{2}+\left|\partial_{2} u\right|^{2}=\frac{1}{2} \mathrm{e}^{v}|\nabla v|^{2}$. Since the function $v$ satisfies

$$
\Delta v=-4 \pi \sum_{s=1}^{P} \delta_{p_{s}}+4 \pi \sum_{s=1}^{N} \delta_{q_{s}} .
$$

In view of (3.16), we see that the energy density (3.6) becomes

$$
\mathrm{e}^{\eta} H=\frac{\mathrm{e}^{v}}{\left(1+\mathrm{e}^{v}\right)^{2}}|\nabla v|^{2}=\Delta \ln \left(1+\mathrm{e}^{v}\right)+4 \pi \sum_{s=1}^{P} \delta_{p_{s}} .
$$

Substituting the expressions $K_{g}=-\frac{1}{2} \mathrm{e}^{-\eta} \Delta \eta$ and (3.17) in (1.6), we conclude that

$$
\eta+16 \pi G\left(\ln \left(1+\mathrm{e}^{v}\right)+\sum_{s=1}^{P} \ln \left|z-p_{s}\right|^{2}\right)
$$

is a harmonic function, which may be assumed to be a constant for convenience. Applying this result and (3.10), we obtain the conformal factor for the gravitational metric explicitly as follows,

$$
\mathrm{e}^{\eta}=g_{0}\left(\prod_{s=1}^{P}\left|z-p_{s}\right|^{2}+|\lambda|^{2} \prod_{s=1}^{N}\left|z-q_{s}\right|^{2}\right)^{-16 \pi G}, \quad z \in \mathbb{C}=\mathbb{R}^{2},
$$

where $g_{0}>0$ is a constant. This metric is everywhere regular. Near infinity, it has the estimate

$$
\mathrm{e}^{\eta}=\mathrm{O}\left(|z|^{-32 P \pi G}\right)
$$

due to (3.13). This estimate implies, when $16 \pi P G<1$, the surface $(S, g)$ has a conical singularity at infinity with the deficit angle $\delta=32 \pi^{2} P G$, and, the surface 
$(S, g)$ is geodesically complete if and only if $P \leq 1 / 16 \pi G$. Of course, as a result of the Einstein equation (1.6), the total curvature is quantized according to

$$
\int_{S} K_{g} \mathrm{~d} V_{g}=32 \pi^{2} G P
$$

Existence of Cosmic Strings in Compact Setting. We now consider the system (3.3) for which the unknown surface $S$ is compact. Using the Gauss-Bonnet theorem (the special case of (1.2) when $n=1$ ), namely,

$$
\int_{S} K_{g} \mathrm{~d} V_{g}=2 \pi \chi(S)=4 \pi(1-k)
$$

where $k$ gives the genus of $S$, the first equation in (3.3), and (2.4), we see that $k=0$, hence $S$ is topologically a 2 -sphere, unless there is no string, $N=0$, for which $k=1$ and $S$ is a flat torus. Therefore, for the nontrivial case where $N>0$ and $k=0$, integrating the first equation in (3.3) and inserting (2.4), we arrive at the quantization condition for the Newton constant $G$,

$$
G=\frac{1}{2 \pi N}
$$

We are interested in the existence of $N$ prescribed cosmic string solutions of the system (3.3) over $S=S^{2}$ subject to the condition (3.23). We state our results $[81,82]$ as follows.

Theorem 3.2.. A necessary condition for the existence of $N(N \geq 1)$ string solutions to the system (3.3) over a compact surface $S$ is that $S$ is topologically a 2-sphere, $S^{2}$, and that the Newton constant $G$ satisfies the quantization condition (3.23). Under this condition, for any points $p_{1}, \cdots, p_{\ell} \in S^{2}$ and integers $n_{1}, \cdots, n_{\ell} \in \mathbb{N}$ with $n_{1}+\cdots+n_{\ell}=N$ and

$$
n_{1}<\frac{1}{2} N, \cdots, n_{\ell}<\frac{1}{2} N
$$

the system (3.3) has a solution triplet $(g, u, A)$ so that it defines an appropriate Hermitian line bundle $\xi$ over $S^{2}$ with the first Chern class $c_{1}(\xi)=N$ and the zeros of the Higgs cross-section $u$ are exactly $p_{1}, \cdots, p_{\ell}$ with the respective multiplicities $n_{1}, \cdots, n_{\ell}$. In particular, when $N \geq 3$, the system (3.3) has an $N$-string solution so that the Higgs field $u$ has simple zeros at the $N$ distinctly prescribed locations on $S^{2}$. When $N=2 N_{0}\left(N_{0} \geq 1\right)$ is an even integer, the system (3.3) has an $N$ string solution so that the centers of the strings are at the north and south poles of $S^{2}$ and there are exactly $N_{0}$ strings at each of these two poles. In particular, there exists a 2-string solution with strings located at the opposite poles. Finally, 
the system (3.3) does not have any $N$-string solution so that the strings are all superimposed at one point on $S^{2}$ and the field configuration is symmetric about this point. In particular, there does not exist any symmetric 1-string solution at all.

Interpretation of the Quantized Newton Constant. The condition (3.23) seems unnatural since it quantizes the Newton constant and needs some interpretation. In fact, such a condition comes from our compact surface setting, $S=S^{2}$, which can be removed when we delete a point from $S^{2}$ to return to a noncompact, $S \backslash\{$ one point $\}=\mathbb{R}^{2}$, setting. When we do this, the point to be deleted may or may not be a string point. In case of a string point, we may delete a string carrying a local string (winding) number up to $N / 2$ according to Theorem 3.2. Consequently, this procedure produces $N$-string solutions over $\mathbb{R}^{2}$ under the condition

$$
\frac{1}{4 \pi G} \leq N \leq \frac{1}{2 \pi G}
$$

Of course, the gravitational metric thus generated over $\mathbb{R}^{2}$ is not complete. Note that these solutions over $\mathbb{R}^{2}$ belong to a different category from and complement those obtained in Theorem 3.1 characterized by $|u|=1$ at infinity.

Issues of Positive Curvature and Energy Lower Bounds in Three Dimensions. At this point, it may be interesting to recall that similar positivity conditions also appear in the Positive Energy Theorem [59, 62, 63, 64, 65, 76] and in the Penrose Conjecture [60] in general relativity. In this context, one is interested in an asymptotically Euclidean space-like hypersurface $(M, g)$ embedded in a four-dimensional Lorentzian spacetime whose metric tensor satisfies the Einstein equations (1.1) for which the energy-momentum tensor $T_{\mu \nu}$ is subject to a physically motivated condition, called the dominant energy condition, which implies that the pointwise energy density $H=T_{00}$ is nonnegative and is in fact bounded from below by the magnitude of the pointwise angular momentum,

$$
H=T_{00} \geq \sqrt{\sum T_{0 j}^{2}} .
$$

The hypersurface $(M, g)$ is said to be asymptotically Euclidean if there is a compact subset $K$ in $M$ such that $M \backslash K$ is the union of finitely many "ends" of $M$ so that each end is diffeomorphic to $\mathbb{R}^{3} \backslash B_{r}$ where $B_{r}$ denotes the ball of radius $r>0$ centered at the origin. Under this diffeomorphism, the metric $g_{j k}$ of $M$ at 
each end can be represented near infinity by

$$
\begin{aligned}
g_{j k}(x) & =\delta_{j k}+a_{j k}(x), \quad x \in \mathbb{R}^{3} \backslash B_{r}, \\
(3.27) a_{j k}(x) & =\mathrm{O}\left(|x|^{-1}\right), \quad \partial_{\ell} a_{j k}(x)=\mathrm{O}\left(|x|^{-2}\right), \quad \partial_{\ell} \partial_{m} a_{j k}(x)=\mathrm{O}\left(|x|^{-3}\right),
\end{aligned}
$$

and the second fundamental form $\left(h_{j k}\right)$ there satisfies similar asymptotic estimates

$$
h_{j k}(x)=\mathrm{O}\left(|x|^{-2}\right), \quad \partial_{\ell} h_{j k}(x)=\mathrm{O}\left(|x|^{-3}\right), \quad x \in \mathbb{R}^{3} \backslash B_{r} .
$$

Without loss of generality, we assume only one end for convenience. According to Arnowitt, Deser, and Misner [6], the total energy $E$ and the momentum $P_{\ell}$ can be defined as the limits of integral fluxes

$$
\begin{aligned}
E & =\frac{1}{16 \pi} \lim _{r \rightarrow \infty} \int_{\partial B_{r}}\left(\partial_{j} g_{j k}-\partial_{k} g_{j j}\right) \nu^{k} \mathrm{~d} \sigma_{r}, \\
P_{\ell} & =\frac{1}{8 \pi} \lim _{r \rightarrow \infty} \int_{\partial B_{r}}\left(h_{\ell k}-\delta_{\ell k} h_{j j}\right) \nu^{k} \mathrm{~d} \sigma_{r},
\end{aligned}
$$

where $\mathrm{d} \sigma_{r}$ is the area element of $\partial B_{r}$ and $\nu$ denotes the outnormal vector to $\partial B_{r}$. The Positive Energy Theorem [59, 62, 63, 64, 76] states that the total energy (3.29) is bounded from below by the total momentum (3.30) by

$$
E \geq|P|
$$

and that $E=0$ if and only if $(M, g)$ is the Euclidean space $\left(\mathbb{R}^{3}, \delta\right)$. In the special case when the second fundamental form $\left(h_{j k}\right)$ vanishes identically, $P \equiv 0$, the energy $E$ is called the total mass or the ADM mass, $m_{\mathrm{ADM}}$, which is always nonnegative, $m_{\mathrm{ADM}} \geq 0$. The Positive Mass Theorem [65] states that

$$
m_{\mathrm{ADM}}>0
$$

unless the hypersurface $(M, g)$ is the Euclidean space $\left(\mathbb{R}^{3}, \delta\right)$.

Simply put, the above theorems imply that no energy or mass means no geometry or no gravitation.

Note that, using the Einstein equations (1.1), one may relate the scalar curvature $R_{g}$ of $(M, g)$ to the energy density $T_{00}$ by

$$
R_{g}+\left(h_{k}^{k}\right)^{2}-h_{j}^{k} h_{k}^{j}=16 \pi G T_{00} .
$$

Thus, as a consequence of the dominant energy condition (see (3.26)), the vanishing of the second fundamental form naturally leads to the positivity condition 
Geometry, Topology, and Gravitation Synthesized by Cosmic Strings 753

for the scalar curvature,

$$
R_{g} \geq 0
$$

which is comparable with our condition for the Gauss curvature, $K_{g} \geq 0$, in the context of cosmic strings.

Naturally, one would hope to bound $m_{\mathrm{ADM}}$ away from zero by some physical information in a gravitational system. For example, one may start from considering an isolated blackhole of mass $m_{\mathrm{BH}}>0$ whose spacetime metric is known to be given by the Schwarzschild line element in terms of the spherical coordinates $(\theta, \phi, \rho)$ as

$$
\mathrm{d} s^{2}=\left(1-\frac{2 G m_{\mathrm{BH}}}{\rho}\right) \mathrm{d} t^{2}-\left(1-\frac{2 G m_{\mathrm{BH}}}{\rho}\right)^{-1} \mathrm{~d} \rho^{2}-\rho^{2}\left(\mathrm{~d} \theta^{2}+\sin ^{2} \theta \mathrm{d} \phi^{2}\right)
$$

It can be checked that the spatial slice at any fixed $t$ has the property that its second fundamental form vanishes and that its ADM mass is the same as the blackhole mass $m_{\mathrm{BH}}$. In this case, the singular surface or the event horizon, $\Sigma$, of the blackhole is a sphere of radius $r_{s}=2 G m_{\mathrm{BH}}$ whose surface area has the value

$$
\operatorname{Area}(\Sigma)=4 \pi r_{s}^{2}=16 \pi G^{2} m_{\mathrm{BH}}^{2} \cdot
$$

The Penrose Conjecture [60] states that the total energy $E$ of the spacetime defined in (3.29) is bounded from below by the total surface area of its apparent horizon $\Sigma$, which coincides with the event horizon in the case of a Schwarzschild blackhole, by

$$
16 \pi G^{2} E^{2} \geq \operatorname{Area}(\Sigma)
$$

In the special case when the second fundamental form of the hypersurface $M$ vanishes, (3.37) becomes

$$
16 \pi G^{2} m_{\mathrm{ADM}}^{2} \geq \operatorname{Area}(\Sigma)
$$

which is referred to as the Riemannian Penrose Inequality, for which the lower bound may be saturated only in the Schwarzschild limit [17, 18, 19, 35, 36].

Comments and Comparisons. We have seen that, both in the 2-dimensional cosmic string setting and in the 3-dimensional Positive Energy Theorem and the Penrose Conjecture settings, the condition that the respective Gauss curvature 
and scalar curvature are positive-valued follows as a consequence of the positiveness of the coupled energy density of the matter sector and plays an important role. In the cosmic string setting, the total curvature may be viewed in the place of the ADM energy or ADM mass and is bounded from below by a quantity related to the total string number, which is the energy of the self-dual string solutions and may be comparable with the energy of the Schwarzschild metric. There are obviously several significant differences, though, in the context of cosmic strings. The first one is that the total energy or total curvature lower bound is instead given by a topological invariant at the bundle level rather than a geometric quantity at the spacetime level. The second one is that the cosmic string metrics can no longer be asymptotically Euclidean. In fact, when restricted to a complete metric, the presence of cosmic strings necessarily makes the infinity of the 2-surface that hosts the strings conical and the deficit angle of the conical infinity is directly proportional to the total string number. Besides, when the total string number is large, the string metric may even become noncomplete. The third one is that, in the context of cosmic strings, a fully coupled system consisting of the Einstein equations and the equations of motion of the matter-gauge sector which determines the energy-momentum tensor $T_{\mu \nu}$ on the right-hand side of the Einstein equations needs to be considered, reflecting the idea that gravitation and other physical interactions are coupled.

\section{Coexisting Cosmic Strings And Antistrings}

Strings and Antistrings Generated from Zeros and Poles. In the explicit construction of Comtet and Gibbons [27] of the multiple string metric induced from the $\sigma$-model or the harmonic maps from $\mathbb{R}^{2}$ to $S^{2}$ discussed in the previous section, the total energy is proportional to the total number of poles of the complex function $u$, which is also the topological degree, of the harmonic map $\phi$. Schroers $[66,67]$ extended the $\sigma$-model to include a gauge field. Within this gauged $\sigma$ model, the zeros and poles of $u$ plays equal roles $[84,85]$ as seen in Section 2, which give rise to vortices and antivortices, so that the total energy is proportional to the total numbers of zeros and poles of $u$. Thus, it is natural that we may expect to produce cosmic strings and antistrings using these vortices and antivortices when we consider the presence of gravity as well as that for the Abelian 
Geometry, Topology, and Gravitation Synthesized by Cosmic Strings 755

Higgs model as in Section 3. Therefore, we are led to the coupled system

$$
\begin{aligned}
K_{g} & =8 \pi G H(u, A), \quad(H \text { is as defined in }(2.7)) \\
D_{A} u \pm \mathrm{i} *_{g} D_{A} u & =0, \\
F_{A} & = \pm *_{g} \frac{1-|u|^{2}}{1+|u|^{2}},
\end{aligned}
$$

which describes the interaction of gravity and electromagnetism. Our existence theorem $[84,85]$ concerning the solutions representing a prescribed distribution of cosmic strings and antistrings for the system (4.1) when the underlying 2-surface $(S, g)$ is noncompact but complete can be stated as follows.

Theorem 4.1.. For any prescribed points $q_{1}, \cdots, q_{N} \in S$ and $p_{1}, \cdots, p_{P} \in S$ (which is necessarily diffeomorphic to $\mathbb{R}^{2}$ ), the system (4.1) has a finite-energy solution $(g, u, A)$ so that $q_{1}, \cdots, q_{N}$ are the zeros and $p_{1}, \cdots, p_{P}$ are the poles of $u,|u|=1$ at the infinity of $S$, and $g$ is a complete metric for $(S, g)$ if and only if the total string number $N$, the total antistring number $P$, and the Newton constant $G$ satisfy

$$
N+P \leq \frac{1}{8 \pi G}
$$

In this case the magnetic flux is quantized according to $\Phi=\int_{S} * F_{A} d V_{g}=$ $2 \pi(N-P)$ and the total curvature and matter-gauge energy assume their minimum quantized values

$$
\int_{S} K_{g} d V_{g}=16 \pi^{2} G(N+P), \quad \int_{S} H(u, A) d V_{g}=2 \pi(N+P) .
$$

Besides, the surface $S$ has a conical singularity at infinity with a deficit angle $\delta=16 \pi^{2} G(N+P)$. If $N=P=0$, the pair $(u, A)$ is gauge-equivalent to the trivial pair $u=1, A=0$ and $(S, g)$ is globally conformal to the standard Euclidean plane $\left(\mathbb{R}^{2}, \delta\right)$.

Strings and Antistrings in Compact Setting. We shall elaborate in more detail on the case when $S$ is a compact surface. As before, it is seen that $S$ must be a 2-sphere. Hence, (1.6) and (2.11) lead to

$$
4 \pi=\int_{S} K_{g} \mathrm{~d} V_{g}=8 \pi G \int_{S} H(u, A) \mathrm{d} V_{g}=16 \pi^{2} G(N+P) .
$$

Consequently, the Newton constant $G$ is again quantized according to

$$
G=\frac{1}{4 \pi(N+P)} \text {. }
$$


For the system of equations (4.1), our main results [88] are summarized as follows.

Theorem 4.2.. Consider the coupled equations (4.1) formulated over a complex line bundle $\xi \rightarrow S$ for which the unknowns $u$ is a cross-section, $A$ is a connection 1-form of the bundle, and the metric $g$ on $S$ is to be determined through its Gauss curvature.

(i) Any solution triplet $(u, A,(S, g))$ so that $u$ has no zeros nor poles on $S$ is gauge-equivalent to a trivial solution for which $\xi \rightarrow S$ is a trivial bundle, $u \equiv 1$, $A \equiv 0$, and $(S, g)$ is a flat 2-torus, which implies the absence of gravitation.

(ii) A solution triplet $(u, A,(S, g))$ of the system (4.1) is characterized by the finite sets of zeros and poles of integral multiplicities of the scalar field u. Moreover, the presence of any zero or pole implies that $S$ is topologically a 2-sphere, $S \approx S^{2}$.

(iii) A necessary condition for the existence of a solution to (4.1) with $N$ zeros and $P$ poles is that the Newton gravitational constant $G$ satisfies the quantization condition (4.5) which will be assumed throughout the rest of the statement of this theorem.

(iv) For a solution triplet $(u, A,(S, g))$, the magnetic field $B=* F_{A}$ assumes its global maximum and minimum values at the zeros, $q$, and poles, $p$, of $u$, respectively, which define the opposite magnetic penetration strengths through the formulas $B(q)=1, B(p)=-1$. In other words, the zeros and poles give rise to cosmic strings and antistrings of opposite magnetic or vorticity excitations.

(v) For a solution triplet $(u, A,(S, g))$ with $N$ zeros and $P$ poles, the magnetic flux and total energy are given respectively by the quanta

$$
\Phi=\int_{S} * F_{A} d V_{g}=2 \pi(N-P), \quad E=\int_{S} H(u, A) d V_{g}=2 \pi(N+P) .
$$

(vi) For any finite sets of prescribed points $Q=\{q\}$ and $P=\{p\}$ on $S$ and the corresponding sets of positive integers $\left\{m_{q} \mid q \in Q\right\}$ and $\left\{n_{p} \mid p \in P\right\}$, the system (4.1) has a solution $(u, A,(S, g))$ so that the sets of zeros and poles of $u$ are $Q$ and $P$ with the respectively assigned multiplicities $\left\{m_{q} \mid q \in Q\right\}$ and $\left\{n_{p} \mid p \in P\right\}$, provided that

$$
m_{q}<\frac{1}{2}(N+P), \quad q \in Q, \quad n_{p}<\frac{1}{2}(N+P), \quad p \in P,
$$


Geometry, Topology, and Gravitation Synthesized by Cosmic Strings 757

where $N=\sum_{q \in Q} m_{q}$ and $P=\sum_{p \in P} n_{p}$. In particular, for any prescribed $N$ distinct points $\left\{q_{1}, q_{2}, \cdots, q_{N}\right\}$ and $P$ distinct points $\left\{p_{1}, p_{2}, \cdots, p_{P}\right\}$ with $N+$ $P \geq 3$, the system (4.1) has a solution $(u, A,(S, g))$ so that the points $q$ 's and $p$ 's are the single zeros and poles of $u$, respectively.

(vii) Consider symmetric solutions only.

(a) Existence: If $N=2 N_{0}$ is even and $P=0$, there is a solution representing $N_{0}$ zeros at the north and $N_{0}$ zeros at the south poles of $S^{2}$; if $N=0$ and $P=2 P_{0}$ is even, there is a solution representing $P_{0}$ poles at the north and $P_{0}$ poles at the south poles of $S^{2}$; if $N=P \neq 0$, there is a solution representing $N$ zeros and $P$ poles at the two opposing poles of $S^{2}$.

(b) Nonexistence: There is no solution for which all zeros or poles are clustered at a single point on $S^{2}$; there is no solution for which there are different numbers of zeros or poles at the two opposing poles of $S^{2}$; in particular, there is no solution when $N+P=1$.

Analogous to the discussion made for the cosmic strings generated in the Abelian Higgs model, we may use the $S^{2}$-setting solutions obtained in Theorem 4.2 to produce solutions representing prescribed $N$ strings and $P$ antistrings in $\mathbb{R}^{2}$ under the condition

$$
\frac{1}{8 \pi G} \leq N+P \leq \frac{1}{4 \pi G}
$$

Again, these solutions give rise to noncomplete gravitational metrics and complement those obtained in Theorem 4.1 under the condition (4.2).

Reduction to Nonlinear PDE. In what follows, we present the nonlinear elliptic equation associated with our existence problem for strings and antistrings. For simplicity, we use the notation $Q=\left\{q_{1}, q_{2}, \cdots, q_{N}\right\}$ and $P=\left\{p_{1}, p_{2}, \cdots, p_{P}\right\}$ over $S=S^{2}$, where we use repeated points to represent their multiplicities as zeros and poles of $u$, respectively.

We will look for a solution $(u, A, g)$ of the system (4.1) so that the sets of points $Q$ and $P$ are the sets of zeros (strings) and poles (antistrings) of $u$. Set $v=\ln |u|^{2}$. Then the last two equations of (4.1) lead to the following single equation over the unknown gravitational metric $g$,

$$
\Delta_{g} v=2\left(\frac{\mathrm{e}^{v}-1}{\mathrm{e}^{v}+1}\right)+4 \pi \sum_{q \in Q} \delta_{q}-4 \pi \sum_{p \in P} \delta_{p},
$$


where $\delta_{p}$ is the Dirac distribution over $(S, g)$ concentrated at a point $p \in S$. Below, we will see that the first equation in (4.1) may be resolved exactly in terms of $v$ as in the Comtet-Gibbons construction.

In fact, using the identity $\left|D_{A} u\right|^{2}=\frac{1}{2} \mathrm{e}^{v}|\nabla v|^{2}$, local coordinates, and local conformality $g=\mathrm{e}^{\eta} \delta$, we see that the Hamiltonian (2.7) becomes

$$
\begin{aligned}
H(u, A) & =\left(\frac{1-|u|^{2}}{1+|u|^{2}}\right)\left(*_{g} F_{A}\right)+\frac{2}{\left(1+|u|^{2}\right)^{2}}\left(*_{g}\left[D_{A} u \wedge *_{g} \overline{D_{A} u}\right]\right) \\
& =\mathrm{e}^{-\eta}\left(\frac{\Delta v}{2}\left[\frac{\mathrm{e}^{v}-1}{\mathrm{e}^{v}+1}\right]+\frac{\mathrm{e}^{v}|\nabla v|^{2}}{\left(\mathrm{e}^{v}+1\right)^{2}}\right) \\
& =\mathrm{e}^{-\eta} \Delta\left(\ln \left(1+\mathrm{e}^{v}\right)-\frac{1}{2} v\right), \quad \text { away from } P \cup Q .
\end{aligned}
$$

Returning to a general coordinate system, we obtain from (4.10) the expression

$$
H(u, A)=\Delta_{g}\left(\ln \left(1+\mathrm{e}^{v}\right)-\frac{1}{2} v\right), \quad \text { away from } P \cup Q .
$$

Review the local behavior of $v=\ln |u|^{2}$ near the poles and zeros of $u$. We see that the right-hand side of (4.11) generates a singular source term $-2 \pi \delta_{q}$ at a single zero of $u$ and a term $-2 \pi \delta_{p}$ at a single pole of $u$. Since $H(u, A)$ is regular, we obtain from (4.11) the global expression

$$
H(u, A)=\Delta_{g}\left(\ln \left(1+\mathrm{e}^{v}\right)-\frac{1}{2} v\right)+2 \pi \sum_{q \in Q} \delta_{q}+2 \pi \sum_{p \in P} \delta_{p} \quad \text { on } S .
$$

On the other hand, recall that, if we assume the unknown gravitational metric $g$ to be conformal to a known background metric $g_{0}$, then

$$
g=\mathrm{e}^{\eta} g_{0}, \quad \Delta_{g}=\mathrm{e}^{-\eta} \Delta_{g_{0}}, \quad-\Delta_{g_{0}} \eta+2 K_{g_{0}}=2 K_{g} \mathrm{e}^{\eta} .
$$

Consequently, (4.9) becomes

$$
\Delta_{g_{0}} v=2 \mathrm{e}^{\eta}\left(\frac{\mathrm{e}^{v}-1}{\mathrm{e}^{v}+1}\right)+4 \pi \sum_{q \in Q} \delta_{q}-4 \pi \sum_{p \in P} \delta_{p},
$$

Note that the Dirac distributions are now over the known surface $\left(S, g_{0}\right)$. Inserting (4.12) and (4.13) into the first equation in (4.1), we get

$$
\Delta_{g_{0}}\left(\frac{\eta}{16 \pi G}+\ln \left(1+\mathrm{e}^{v}\right)-\frac{1}{2} v\right)=\frac{K_{0}}{8 \pi G}-2 \pi \sum_{q \in Q} \delta_{q}-2 \pi \sum_{p \in P} \delta_{p} \quad \text { on } S .
$$

Since $S$ is topologically a 2 -sphere, we have $\int_{S} K_{0} \mathrm{~d} V_{g_{0}}=4 \pi$. Using this and (4.5), we see that the right-hand side of (4.15) yields a zero value when integrated 
over $\left(S, g_{0}\right)$ (the integrability condition). Hence, there is a unique function $V_{0}$ (uniqueness up to a translation by a constant) so that

$$
\frac{\eta}{8 \pi G}+2 \ln \left(1+\mathrm{e}^{v}\right)-v=-V_{0}+c
$$

where $V_{0}$ is a solution of the equation

$$
\Delta_{g_{0}} V_{0}=-\frac{K_{0}}{4 \pi G}+4 \pi \sum_{q \in Q} \delta_{q}+4 \pi \sum_{p \in P} \delta_{p}
$$

and $c$ is an arbitrary constant. The function $V_{0}$ has the following property that, if for each pole $p$ (or zero $q$ ) of multiplicity $n$ on $S$, letting $\left(U,\left(x^{j}\right)\right)$ be a small isothermal coordinate chart around $p$ (or $q$ ), then $V_{0}$ can be decomposed as

$$
V_{0}(x)=w_{0}(x)+n \ln |x|^{2},
$$

where $w_{0}$ is smooth on $U$. Therefore, we have obtained the unknown conformal exponent $\eta$ in terms of the unknown $v$ so that (4.16) gives us a resolution of the reduced Einstein equation (the first equation in (4.1).

Finally, inserting (4.16) into (4.14), we arrive at the following single governing equation

$$
\Delta_{g_{0}} v=2 \lambda \mathrm{e}^{-a V_{0}}\left(\frac{\mathrm{e}^{v}}{\left(1+\mathrm{e}^{v}\right)^{2}}\right)^{a}\left(\frac{\mathrm{e}^{v}-1}{\mathrm{e}^{v}+1}\right)+4 \pi \sum_{q \in Q} \delta_{q}-4 \pi \sum_{p \in P} \delta_{p},
$$

where $\lambda>0$ is an adjustable constant and $a=8 \pi G$. In view of (4.5), the constant $a$ depends only on the sum of $N$ and $P: a=2 /(N+P)$. Therefore, we have seen that our existence problem for $N$ prescribed strings (zeros) and $P$ prescribed antistrings (poles) over $S$ is reduced to the solvability of (4.19).

Solution via Chern-Simons Equation and Constrained Variational Approach. Due to its structure, we may relate the solvability of (4.19) to that of the well-known Abelian Chern-Simons vortex equation

$$
\Delta_{g_{0}} v=\lambda \mathrm{e}^{v}\left(\mathrm{e}^{v}-1\right)+4 \pi\left(\sum_{q \in Q} \delta_{q}+\sum_{p \in P} \delta_{p}\right)
$$

arising in condensed-matter physics [29, 34, 37]. Indeed, we can use the solution of (4.20) to generate an approximate solution of (4.19) which is obtained as a solution of a perturbed form of (4.19). We then pass to the zero perturbation limit to recover a solution of (4.19) itself. In this way, the resolution of (4.19) 
boils down to the resolution of (4.20). To see the detailed structure of (4.20), we use $|S|$ to denote the total surface area of $\left(S, g_{0}\right)$ and let $v_{0}$ be any solution of

$$
\Delta_{g_{0}} v_{0}=-\frac{4 \pi(N+P)}{|S|}+4 \pi\left(\sum_{q \in Q} \delta_{q}+\sum_{p \in P} \delta_{p}\right) .
$$

With $v=v_{0}+w$, we convert (4.20) into

$$
\Delta_{g_{0}} w=\lambda \mathrm{e}^{v_{0}+w}\left(\mathrm{e}^{v_{0}+w}-1\right)+\frac{4 \pi(N+P)}{|S|} .
$$

Completing square on the right-hand side of (4.22) and integrating over $S$, we get

$$
\int_{S}\left(\mathrm{e}^{v_{0}+w}-\frac{1}{2}\right)^{2} \mathrm{~d} V_{g_{0}}=\frac{|S|}{4}-\frac{4 \pi(N+P)}{\lambda} .
$$

Hence it is necessary that $\lambda$ satisfies $\lambda>16 \pi(N+P) /|S|$. In [21], it is shown that there is a critical number $\lambda_{c} \geq 16 \pi(N+P) /|S|$, so that whenever $\lambda>\lambda_{c}$, the equation (4.22) has a solution. We now explain why a solution of (4.22) exists whenever $\lambda$ is sufficiently large through a variational approach which involves minimization subject to an inequality constraint [87] and is of independent interest.

To save notation somewhat, we rewrite the unknown $w$ as $v$ again, suppress $N+P$ as $N, p$ and $q$ simply as $p$, and set $U=\mathrm{e}^{v_{0}}$. Then (4.22) becomes

$$
\Delta v=\lambda U \mathrm{e}^{v}\left(U \mathrm{e}^{v}-1\right)+\frac{4 \pi N}{|S|} .
$$

The function $U \geq 0$ is smooth since $v_{0}$ behaves like $\ln |x-p|^{2}$ near the prescribed vortex point $p$ (see (4.18)).

We shall work on the standard Sobolev space $S=W^{1,2}(S)$. Then

$$
X=\left\{v \in S \mid \int_{S} v=0\right\}
$$

is a closed subspace of $S$ and $S=\mathbb{R} \oplus X$. That is, for any $v \in S$, there is a unique number $c \in \mathbb{R}$ and $v^{\prime} \in X$ so that

$$
v=c+v^{\prime}
$$

Here and in the sequel, we skip expressing the surface element $\mathrm{d} V_{g_{0}}$ in an integral when there is no risk of confusion. 
Geometry, Topology, and Gravitation Synthesized by Cosmic Strings 761

Suppose that $v \in S$ given in (4.25) satisfies (4.23). Then

$$
\mathrm{e}^{2 c} \int_{S} U^{2} \mathrm{e}^{2 v^{\prime}}-\mathrm{e}^{c} \int_{S} U \mathrm{e}^{v^{\prime}}+\frac{4 \pi N}{\lambda}=0 .
$$

Of course (4.26) is a quadratic equation in $t=\mathrm{e}^{c}$ which has a solution if and only if

$$
\left(\int_{S} U \mathrm{e}^{v^{\prime}}\right)^{2}-\frac{16 \pi N}{\lambda} \int_{S} U^{2} \mathrm{e}^{2 v^{\prime}} \geq 0 .
$$

In this case we may choose $c=c\left(v^{\prime}\right)$ in (4.26) to satisfy

$$
\mathrm{e}^{c}=\frac{\int_{S} U \mathrm{e}^{v^{\prime}}+\sqrt{\left(\int_{S} U \mathrm{e}^{v^{\prime}}\right)^{2}-\frac{16 \pi N}{\lambda} \int_{S} U^{2} \mathrm{e}^{2 v^{\prime}}}}{2 \int_{S} U^{2} \mathrm{e}^{2 v^{\prime}}} .
$$

With $v^{\prime}$ satisfying (4.27) and $c$ given by (4.28), we define a functional $I$ on $X$ by the expression

$$
I\left(v^{\prime}\right)=\int_{S}\left\{\frac{1}{2}\left|\nabla v^{\prime}\right|^{2}+\frac{\lambda}{2} U^{2} \mathrm{e}^{2 c+2 v^{\prime}}-\lambda U \mathrm{e}^{c+v^{\prime}}\right\}+4 \pi N c .
$$

Set $A=\left\{v^{\prime} \in X \mid v^{\prime}\right.$ satisfies (4.27) $\}$. Consider the optimization problem

$$
\min \left\{I\left(v^{\prime}\right) \mid v^{\prime} \in A\right\} \text {. }
$$

We shall find some condition under which the problem (4.30) has only interior minimizers.

Lemma 4.3.. For $v^{\prime} \in X$ on the boundary of $A$, namely,

$$
\left(\int_{S} U e^{v^{\prime}}\right)^{2}-\frac{16 \pi N}{\lambda} \int_{S} U^{2} e^{2 v^{\prime}}=0,
$$

we have $I\left(v^{\prime}\right) \geq-4 \pi \ln \lambda-C$ for some constant $C>0$ independent of $\lambda$.

Proof. From (4.28) and (4.31), we obtain

$$
\mathrm{e}^{c}=\frac{\int_{S} U \mathrm{e}^{v^{\prime}}}{2 \int_{S} U^{2} \mathrm{e}^{2 v^{\prime}}}=\frac{8 \pi N}{\lambda \int_{S} U \mathrm{e}^{v^{\prime}}} .
$$

Therefore a simple calculation shows that

$$
I\left(v^{\prime}\right)=-6 \pi N+\frac{1}{2}\left\|\nabla v^{\prime}\right\|_{2}^{2}+4 \pi N c,
$$

where and in the sequel we use \|\|$_{2}$ to denote the usual $L^{2}$-norm on $S$. 
We rewrite (4.32) as

$$
c=\ln 8 \pi N-\ln \lambda-\ln \left(\int_{S} U \mathrm{e}^{v^{\prime}}\right) .
$$

Let $p, q>1$ be conjugate exponents to be determined so that $1 / p+1 / q=1$. In view of the Schwartz inequality and the Trudinger-Moser inequality $[7,8,23$, $53,71]$

$$
\int_{S} \mathrm{e}^{f} \leq C(\varepsilon) \exp \left(\left[\frac{1}{16 \pi}+\varepsilon\right] \int_{S}|\nabla f|^{2}\right), \quad f \in W^{1,2}(S), \quad \int_{S} f=0,
$$

we have the following upper bound for $\ln \left(\int U \mathrm{e}^{v^{\prime}}\right)$,

$$
\begin{aligned}
& \ln \left(\int_{S} U \mathrm{e}^{v^{\prime}}\right) \leq \frac{1}{p} \ln \left(\int_{S} U^{p}\right)+\frac{1}{q} \ln \left(\int_{S} \mathrm{e}^{q v^{\prime}}\right) \\
& \leq \frac{1}{p} \ln \left(\int_{S} U^{p}\right)+q\left(\frac{1}{16 \pi}+\varepsilon\right)\left\|\nabla v^{\prime}\right\|_{2}^{2}+\frac{1}{q} \ln C(\varepsilon) .
\end{aligned}
$$

Using (4.34) and (4.36) in (4.33), we arrive at

$$
I\left(v^{\prime}\right) \geq\left(\frac{1}{2}-4 \pi N q\left[\frac{1}{16 \pi}+\varepsilon\right]\right)\left\|\nabla v^{\prime}\right\|_{2}^{2}-4 \pi N \ln \lambda-C(\varepsilon, q) .
$$

If $N=1$, we can choose suitable $\varepsilon>0$ and $q>1$ above to make the coefficient of the first term on the right-hand side of (4.37) positive. If $N>1$, we need an inequality derived by Nolasco and Tarantello $[57,58]$ for functions in $A$.

In fact, we may rewrite the constraint (4.27) as

$$
\int_{S} U^{2} \mathrm{e}^{2 v^{\prime}} \leq \frac{\lambda}{16 \pi N}\left(\int_{S} U \mathrm{e}^{v^{\prime}}\right)^{2} .
$$

Let $s \in(0,1)$ and $a=1 /(2-s)$. Then $s a+2(1-a)=1$. Since $p=1 / a, q=$ $1 /(1-a)>1$ are conjugate exponents, namely, $1 / p+1 / q=1$, we have in view of (4.38) that

$$
\begin{aligned}
\int_{S} U \mathrm{e}^{v^{\prime}} & =\int_{S}\left(U^{s a} \mathrm{e}^{s a v^{\prime}}\right)\left(U^{2(1-a)} \mathrm{e}^{2(1-a) v^{\prime}}\right) \\
& \leq\left(\int_{S} U^{s} \mathrm{e}^{s v^{\prime}}\right)^{a}\left(\int_{S} U^{2} \mathrm{e}^{2 v^{\prime}}\right)^{1-a} \\
& \leq\left(\frac{\lambda}{16 \pi N}\right)^{1-a}\left(\int_{S} U^{s} \mathrm{e}^{s v^{\prime}}\right)^{a}\left(\int_{S} U \mathrm{e}^{v^{\prime}}\right)^{2(1-a)}
\end{aligned}
$$


Geometry, Topology, and Gravitation Synthesized by Cosmic Strings 763

which reads,

$$
\left(\int_{S} U \mathrm{e}^{v^{\prime}}\right)^{2 a-1} \leq\left(\frac{\lambda}{16 \pi N}\right)^{1-a}\left(\int_{S} U^{s} \mathrm{e}^{s v^{\prime}}\right)^{a}
$$

Consequently, there holds

$$
\begin{aligned}
\int_{S} U \mathrm{e}^{v^{\prime}} & \leq\left(\frac{\lambda}{16 \pi N}\right)^{\frac{1-s}{s}}\left(\int_{S} U^{s} \mathrm{e}^{s v^{\prime}}\right)^{\frac{1}{s}} \\
& \leq\left(\frac{\lambda}{16 \pi N}\right)^{\frac{1-s}{s}}\left(\max _{x \in S}\{U(x)\}\right)\left(\int_{S} \mathrm{e}^{s v^{\prime}}\right)^{\frac{1}{s}} .
\end{aligned}
$$

Applying (4.35) in (4.39), we obtain the following upper bound instead of (4.36),

$$
\ln \left(\int_{S} U \mathrm{e}^{v^{\prime}}\right) \leq C_{1}(\varepsilon, s)+\left(\frac{1}{16 \pi N}+\varepsilon\right) s\left\|\nabla v^{\prime}\right\|_{2}^{2} \quad v^{\prime} \in A,
$$

where $C(\varepsilon, s)>0$ is a constant depending on $\varepsilon$ and $s$. Thus, in place of (4.37), we have

$$
I\left(v^{\prime}\right) \geq\left(\frac{1}{2}-4 \pi N s\left[\frac{1}{16 \pi}+\varepsilon\right]\right)\left\|\nabla v^{\prime}\right\|_{2}^{2}-4 \pi N \ln \lambda-C_{2}(\varepsilon, s) .
$$

Of course, given $N$, we can choose $s$ to make the coefficient of the first term on the right-hand side of (4.41) positive. Therefore the lemma follows.

We now evaluate $I$ at an interior trial point in the admissible set $A$. For convenience, we choose $v^{\prime}=0$ as a trial element.

Lemma 4.4.. Suppose that $\lambda>0$ is sufficiently large so that

$$
\left(\int_{S} U\right)^{2}-\frac{16 \pi N}{\lambda} \int_{S} U^{2}>0
$$

i.e., $v^{\prime}=0$ lies in the interior of $A$. Then there are constants $C_{1}, C_{2}>0$ independent of $\lambda$ so that $I(0) \leq-C_{1} \lambda+C_{2}$.

Proof. Assume that $c_{0}=c$ is given by the expression (4.28) with $v^{\prime}=0$. The expression (4.29) with $c=c_{0}$ and $v^{\prime}=0$ enable us to obtain

$$
I(0)=-\frac{\lambda}{2} \mathrm{e}^{c_{0}} \int_{S} U+4 \pi N\left(c_{0}-\frac{1}{2}\right) .
$$

However, the equation (4.28) says that

$$
\frac{\int_{S} U}{2 \int_{S} U^{2}}<\mathrm{e}^{c_{0}}<\frac{\int_{S} U}{\int_{S} U^{2}} .
$$


Inserting this into (4.42), we obtain

$$
I(0)<-\lambda \frac{\left(\int_{S} U\right)^{2}}{4 \int_{S} U^{2}}+4 \pi N \ln \left(\frac{\int_{S} U}{\int_{S} U^{2}}\right) .
$$

Recall that $U$ is independent of $\lambda$. Therefore the lemma follows.

From the above two lemmas, we see that there is a $\lambda_{0}>0$ so that

$$
I(0)<-1+I\left(w^{\prime}\right), \quad w^{\prime} \in \partial A, \quad \lambda>\lambda_{0} .
$$

So it is hopeful to get an interior minimizer for (4.30). From now on we always assume that $\lambda$ is such that (4.43) holds.

Lemma 4.5.. There are constants $C_{1}, C_{2}>0$ so that

$$
I\left(v^{\prime}\right) \geq C_{1}\left\|\nabla v^{\prime}\right\|_{2}^{2}-C_{2}, \quad v^{\prime} \in A .
$$

Proof. Using (4.27) and (4.28), we have

$$
\mathrm{e}^{c} \geq \frac{8 \pi N}{\lambda}\left(\int_{S} U \mathrm{e}^{v^{\prime}}\right)^{-1} .
$$

As a consequence,

$$
c \geq-\ln \left(\int_{S} U \mathrm{e}^{v^{\prime}}\right)+\ln \left(\frac{8 \pi N}{\lambda}\right)
$$

On the other hand, the two exponential terms in $I\left(v^{\prime}\right)$ (see (4.29)) are easily controlled. In fact, using the Schwarz inequality, we have

$$
\begin{aligned}
\int_{S}\left\{\frac{1}{2} U^{2} \mathrm{e}^{2 c+2 v^{\prime}}-U \mathrm{e}^{c+v^{\prime}}\right\} & =\frac{1}{2} \int_{S}\left(U \mathrm{e}^{c+v^{\prime}}-1\right)^{2}-\frac{1}{2}|S| \\
& \geq-2|S| .
\end{aligned}
$$

Finally, inserting (4.44) and (4.45) into (4.29) and applying the TrudingerMoser inequality (4.35) again as in the proof of Lemma 4.3, we arrive at the conclusion of the lemma.

Lemma 4.6.. The problem (4.30) has a minimizer $v^{\prime}$ which lies in the interior of the admissible set $A$. 
Proof. Let $\left\{v_{n}^{\prime}\right\}$ be a minimizing sequence of (4.30). From Lemma 4.5 and the Poincaré inequality, we see that $\left\{v_{n}^{\prime}\right\}$ is bounded in $X$. Therefore we may assume without loss of generality that $\left\{v_{n}^{\prime}\right\}$ weakly converges to an element of $X$, say, $v^{\prime}$. Since the mapping $X \rightarrow L(\Omega)$ given by $f \mapsto \mathrm{e}^{f}$ is well-defined and compact (see [7]), we know that $v^{\prime} \in A$ and $c\left(v_{n}^{\prime}\right) \rightarrow c\left(v^{\prime}\right)$ as $n \rightarrow \infty$. Applying this observation in (4.29) we see that $v^{\prime}$ is a minimizer of (4.30). Moreover, (4.43) implies

$$
I\left(v^{\prime}\right) \leq-1+\inf \left\{I\left(w^{\prime}\right) \mid w^{\prime} \in \partial A\right\} .
$$

In other words, $v^{\prime}$ belongs to the interior of $A$.

Since our optimization problem is defined on the subspace $X$ of $S$, it is not obvious whether a critical point of $I$ in $X$ gives rise to a solution of the equation (4.24). In the following, we will examine that the composition $c+v^{\prime}$ with $c$ defined by (4.28) indeed is a critical point of $I$ in the full $S$ and thus solves the equation $(4.24)$.

Lemma 4.7.. Let $v^{\prime}$ be the minimizer produced in Lemma 4.6 and the number $c$ defined by (4.28). Then $v=c+v^{\prime}$ is a solution of (4.24).

Proof. In fact, since $v^{\prime}$ is an interior minimizer, the Fréchet derivative of $I$ at $v^{\prime}$ vanishes:

$$
\left[\mathrm{d} I\left(v^{\prime}\right)\right]\left(w^{\prime}\right)=0 \quad \text { for any } w^{\prime} \in X .
$$

It is more convenient to rewrite the above equation in the functional form

$$
\begin{aligned}
& 0=\int_{S}\left\{\nabla v^{\prime} \cdot \nabla w^{\prime}+\lambda\left[U^{2} \mathrm{e}^{2 c\left(v^{\prime}\right)+2 v^{\prime}}-U \mathrm{e}^{c\left(v^{\prime}\right)+v^{\prime}}\right] w^{\prime}\right\} \\
& +\left[D_{w^{\prime}} c\left(v^{\prime}\right)\right] \int_{S}\left\{\lambda\left[U^{2} \mathrm{e}^{2 c\left(v^{\prime}\right)+2 v^{\prime}}-U \mathrm{e}^{c\left(v^{\prime}\right)+v^{\prime}}\right]+\frac{4 \pi N}{|S|}\right\},
\end{aligned}
$$

where the numerical factor in front of the second integral above, i.e.,

$$
D_{w^{\prime}} c\left(v^{\prime}\right)=\left.\frac{\mathrm{d}}{\mathrm{d} t} c\left(v^{\prime}+t w^{\prime}\right)\right|_{t=0},
$$

is the directional derivative of $c$ at $v^{\prime}$ along $w^{\prime}$. On the other hand, in view of the equation (4.26), the second integral above actually vanishes. Thus (4.46) takes the simplified form

$$
\int_{S}\left\{\nabla v^{\prime} \cdot \nabla w^{\prime}+\lambda\left[U^{2} \mathrm{e}^{2 c+2 v^{\prime}}-U \mathrm{e}^{c+v^{\prime}}\right] w^{\prime}\right\}=0 .
$$


Consider the decomposition $L^{2}(S)=\mathbb{R} \oplus Y$ where

$$
Y=\left\{f \in L^{2}(S) \mid \int_{S} f=0\right\} .
$$

Choose a suitable $\sigma \in \mathbb{R}$ such that

$$
\lambda\left(U^{2} \mathrm{e}^{2 c+2 v^{\prime}}-U \mathrm{e}^{c+v^{\prime}}\right)+\sigma \in Y .
$$

Then the relation $X \subset Y$ and (4.47) imply that

$$
\begin{aligned}
0 & =\int_{S}\left\{\nabla v^{\prime} \cdot \nabla w^{\prime}+\left(\lambda\left[U^{2} \mathrm{e}^{2 c\left(v^{\prime}\right)+2 v^{\prime}}-U \mathrm{e}^{c\left(v^{\prime}\right)+v^{\prime}}\right]+\sigma\right) w^{\prime}\right\} \\
& =\int_{S}\left\{\nabla v^{\prime} \cdot \nabla\left(a+w^{\prime}\right)+\left(\lambda\left[U^{2} \mathrm{e}^{2 c\left(v^{\prime}\right)+2 v^{\prime}}-U \mathrm{e}^{c\left(v^{\prime}\right)+v^{\prime}}\right]+\sigma\right)\left(a+w^{\prime}\right)\right\}
\end{aligned}
$$

for any $a \in \mathbb{R}$. Consequently,

$$
\int_{S}\left\{\nabla v^{\prime} \cdot \nabla w+\left(\lambda\left[U^{2} \mathrm{e}^{2 c+2 v^{\prime}}-U \mathrm{e}^{c+v^{\prime}}\right]+\sigma\right) w\right\}=0, \quad \forall w \in S .
$$

This equation implies that $v^{\prime}$ is a smooth solution of

$$
\Delta v^{\prime}=\lambda U \mathrm{e}^{c+v^{\prime}}\left(U \mathrm{e}^{c+v^{\prime}}-1\right)+\sigma .
$$

Integrating (4.48) yields

$$
\lambda \int_{S}\left(U \mathrm{e}^{c+v^{\prime}}-U^{2} \mathrm{e}^{2 c+2 c^{\prime}}\right)=\sigma|S| .
$$

Comparing the above equation with (4.26), we obtain immediately the relation $\sigma|S|=4 \pi N$. Thus, by (4.48), we see that $v=c+v^{\prime}$ solves (4.24) and the existence proof is complete.

Recall that the parameter $\lambda>0$ is a free parameter which comes from the resolution of the first equation in (4.1). Thus we can always assume $\lambda$ to be sufficiently large so that (4.22) has a solution. We may then use this solution to construct a solution of the (4.19) as described earlier. The details of such a construction can be found in [88].

Some Open Issues. An unsolved case is whether there is a solution representing unbalanced $N$ clustered zeros and $P$ clustered poles at the north and south poles, respectively, where $N, P>0$ but $N \neq P$.

Another interesting question to ask is whether a solution representing unbalanced $N$ clustered zeros and $P$ clustered poles at the north and south poles, 
respectively, is necessarily symmetric. In other words, whether any solution to the coupled equations (cf. (4.14) and (4.15)):

$$
\begin{gathered}
\Delta_{g_{0}} v=2 \mathrm{e}^{\eta}\left(\frac{\mathrm{e}^{v}-1}{\mathrm{e}^{v}+1}\right)+4 \pi N \delta_{\mathbf{n}}(x)-4 \pi P \delta_{\mathbf{s}}(x), \\
\Delta_{g_{0}}\left(\frac{\eta}{16 \pi G}+\ln \left(1+\mathrm{e}^{v}\right)-\frac{1}{2} v\right)=\frac{K_{0}}{8 \pi G}-2 \pi N \delta_{\mathbf{n}}(x)-2 \pi P \delta_{\mathbf{s}}(x)
\end{gathered}
$$

over $S^{2}$ is symmetric with respect to the two poles $\mathbf{n}$ and $\mathbf{s}$ of $S^{2}$, where $g_{0}$ is the standard metric on $S^{2}, K_{0}$ is its associated (constant) Gauss curvature, and the Newton constant $G$ satisfies (4.5).

\section{Topology, Geometry, and Gravitation}

In this paper, we have presented studies of cosmic string solutions which are static solutions of the coupled Einstein equations and some matter-gauge equations for which the nontrivial geometry and topology are clustered in a 2dimensional setting involving a complex line bundle over a Riemann surface. The existence of gravitation is equivalent to the existence of cosmic strings which are characterized by the topology of the line bundle, which in turn, determines the topology of the Riemann surface to that of a 2-sphere via the Gauss-Bonnet theorem. The (lower level) Riemann surface is part of the spacetime, called external space, which houses gravitation, and, the (upper level) line bundle is part of the interaction space, called the internal space, which houses other fundamental interactions. We have seen that, in the context of cosmic strings under consideration, we have the equivalence relation

lower level topology $=$ upper level topology $=$ energy $=$ geometry/gravitation .

The key link of the above relation is the reduced Einstein equation (1.6) which directly relates the space curvature to the energy density of the matter-gauge sector. The matter-gauge sector possesses positive energy if and only if there are strings present, or if and only if the associated complex line bundle possesses a nontrivial topology, which immediately implies via (1.6) that the underlying Riemann surface possesses nontrivial geometry/topology and gravity is present. On the other hand, if the underlying Riemann surface houses nontrivial geometry/gravitation, (1.6) implies that the interaction energy of the matter-gauge 
sector will be positive which is equivalent to the presence of strings and a nontrivial line bundle topology. Consequently, in this context, we see that topology, geometry, energy, and gravitation are indeed equivalent. The topological nature of these cosmic strings has earned for themselves the name "topological defects" $[43,44,72,73]$ as vividly described in the popular science article by Gangui [30].

The author's research was supported in part by NSF grant DMS-0406446.

\section{REFERENCES}

[1] A. Actor, Classical solutions of $S U(2)$ Yang-Mills theories, Rev. Mod. Phys. 51, 461-525 (1979).

[2] J. Ambjorn and P. Olesen, Anti-screening of large magnetic fields by vector bosons, Phys. Lett. B 214, 565-569 (1988).

[3] J. Ambjorn and P. Olesen, On electroweak magnetism, Nucl. Phys. B 315, 606-614 (1989).

[4] J. Ambjorn and P. Olesen, A magnetic condensate solution of the classical electroweak theory, Phys. Lett. B 218, 67-71 (1989).

[5] J. Ambjorn and P. Olesen, A condensate solution of the classical electroweak theory which interpolates between the broken and the symmetric phase, Nucl. Phys. B 330, 193-204 (1990).

[6] R. Arnowitt, S. Deser, and C. Misner, Coordinate invariance and energy expressions in general relativity, Phys. Rev. 122, 997-1006 (1961).

[7] T. Aubin, Nonlinear Analysis on Manifolds: Monge-Ampére Equations, Springer, Berlin and New York, 1982.

[8] T. Aubin, Meilleures constantes dans le théorème d'inclusion de Sobolev et un théorème de Fredholm non linéaire pour la transformation conforme de courburne scalaire, J. Funct. Anal. 32, 148-174 (1979).

[9] P. Aviles, Conformal complete metrics with prescribed non-negative Gaussian curvature in $\mathbb{R}^{2}$, Invent. Math. 83, 519-544 (1986).

[10] A. Bahri and J. Coron, Une theorie des points critiques a l'infini pour l'equation de Yamabe et le probleme de Kazdan-Warner, C. R. Acad. Sci. Paris, Ser. I 15, 513-516 (1985).

[11] A. A. Belavin and A. M. Polyakov, Metastable states of two-dimensional isotropic ferromagnets, JETP Lett. 22, 245-247 (1975).

[12] M. S. Berger, On Riemannian structures of prescribed Gaussian curvature for compact 2-manifolds, J. Diff. Geom. 5 (1971) 325-332.

[13] E. B. Bogomol'nyi, The stability of classical solutions, Sov. J. Nucl. Phys. 24, 449-454 (1976).

[14] M. Born and L. Infeld, Foundation of the new field theory, Nature 132, 1004 (1933).

[15] M. Born and L. Infeld, Foundation of the new field theory, Proc. Roy. Soc. A 144, 425-451 (1934). 
[16] S. Bradlow, Vortices in holomorphic line bundles over closed Kähler manifolds, Commun. Math. Phys. 135, 1-17 (1990).

[17] H. L. Bray, Proof of the Riemannian Penrose inequality using the positive mass theorem, J. Diff. Geom. 59, 177-267 (2001).

[18] H. L. Bray, Black holes, geometric flows, and the Penrose inequality in general relativity, Notices Amer. Math. Soc. 49, 1372-1381 (2002).

[19] H. L. Bray and R. M. Schoen, Recent proofs of the Riemannian Penrose conjecture, Current Developments in Mathematics, Int. Press, Somerville, MA, 1999. pp. 1-36.

[20] F. Bethuel, H. Brezis, and F. Helein, Ginzburg-Landau Vortices, Birkhäuser, Boston, 1994.

[21] L. Caffarelli and Y. Yang, Vortex condensation in the Chern-Simons Higgs model: an existence theorem, Commun. Math. Phys. 168, 321-336 (1995).

[22] S. Y. A. Chang and P. Yang, Prescribing Gaussian curvature on $S^{2}$, Acta Math. 159, 215-259 (1987).

[23] S. Y. A. Chang and P. C. Yang, The inequality of Moser and Trudinger and applications to conformal geometry, Comm. Pure Appl. Math. 56, 1135-1150 (2003).

[24] J. Cheeger and D. Gromoll, On the structure of complete manifolds of nonnegative curvature, Ann. of Math. 96 (1972) 413-443.

[25] C.-C. Chen and C.-S. Lin, Sharp estimates for solutions of multi-bubbles in compact Riemann surfaces, Comm. Pure Appl. Math. 55, 728-771 (2002).

[26] W. Chen and C. Li, A necessary and sufficient condition for the Nirenberg problem, Comm. Pure Appl. Math. 48, 657-667 (1995).

[27] A. Comtet and G. W. Gibbons, Bogomol'nyi bounds for cosmic strings, Nucl. Phys. B 299 (1988) 719-733.

[28] Q. Du, M. Gunzburger, and J. Peterson, Analysis and approximation of the GinzburgLandau model of superconductivity, SIAM Rev. 34, 54-81 (1992).

[29] G. Dunne, Self-Dual Chern-Simons Theories, Lecture Notes in Physics, vol. m 36, Springer, Berlin, 1995.

[30] A. Gangui, Superconducting cosmic strings, American Scientists, 88, May-June Issue, 2000.

[31] O. Garcia-Prada, A direct existence proof for the vortex equations over a compact Riemann surface, Bull. London Math. Soc. 26, 88-96 (1994).

[32] V. L. Ginzburg and L. D. Landau, On the theory of superconductivity, in Collected Papers of L. D. Landau (edited by D. Ter Haar), pp. 546-568, Pergamon, New York, 1965.

[33] Z.-C. Han, Prescribing Gaussian curvature on $S^{2}$, Duke Math. J. 61, 679-703 (1990).

[34] J. Hong, Y. Kim and P.-Y. Pac, Multivortex solutions of the Abelian Chern-Simons-Higgs theory, Phys. Rev. Lett. 64, 2330-2333 (1990).

[35] G. Huisken and T. Ilmanen, The Riemannian Penrose inequality, Int. Math. Res. Not. 20, 1045-1058 (1997).

[36] G. Huisken and T. Ilmanen, The inverse mean curvature flow and the Riemannian Penrose inequality, J. Diff. Geom. 59, 353-437 (2001).

[37] R. Jackiw and E. J. Weinberg, Self-dual Chern-Simons vortices, Phys. Rev. Lett. 64, 23342337 (1990).

[38] A. Jaffe and C. H. Taubes, Vortices and Monopoles Birkhäuser, Boston, 1980. 
[39] J. L. Kazdan, Prescribing the Curvature of a Riemannian Manifold, Regional Conf. Series in Math. 57, Amer. Math. Soc., Providence, 1985.

[40] J. L. Kazdan and F. W. Warner, Integrability conditions for $\Delta u=k-K \mathrm{e}^{2 u}$ with applications to Riemannian geometry, Bull. Amer. Math. Soc. 77, 819-823 (1971).

[41] J. L. Kazdan and F. W. Warner, Curvature functions for compact 2-manifolds, Ann. Math. 99, 14-47 (1974).

[42] J. L. Kazdan and F. W. Warner, Curvature functions for open 2-manifolds, Ann. Math. 99, 203-219 (1974).

[43] T. W. B. Kibble, Some implications of a cosmological phase transition, Phys. Rep. 69, 183-199 (1980).

[44] T. W. B. Kibble, Cosmic strings - an overview, in The Formation and Evolution of Cosmic Strings, ed. G. Gibbons, S. Hawking, and T. Vachaspati, Cambridge University Press, pp. 3-34, 1990.

[45] M.-H. Kiessling, Electromagnetic field theory without divergence problems. I. The Born legacy, J. Statist. Phys. 116, 1057-1122 (2004).

[46] M.-H. Kiessling, Electromagnetic field theory without divergence problems. II. A least invasively quantized theory, J. Statist. Phys. 116, 1123-1159 (2004).

[47] Y. Li, Harnack type inequality: the method of moving planes Commun. Math. Phys. 200, 421-444 (1999).

[48] F. Lin, Complex Ginzburg-Landau equations and dynamics of vortices, filaments, and codimension-2 submanifolds, Comm. Pure Appl. Math. 51, 385-441 (1998).

[49] F. Lin and Y. Yang, Gauged harmonic maps, Born-Infeld electromagnetism, and magnetic vortices, Comm. Pure Appl. Math. LVI, 1631-1665 (2003).

[50] B. Linet, A vortex-line model for a system of cosmic strings in equilibrium, Gen. Relat. Grav. 20 (1988) 451-456.

[51] B. Linet, On the supermassive $U(1)$ gauge cosmic strings, Class. Quantum Grav. 20, L75L79 (1990).

[52] R. McOwen, Conformal metrics in $\mathbb{R}^{2}$ with prescribed Gaussian curvature and positive total curvature, Indiana U. Math. J. 34, 97-104 (1984).

[53] J. Moser, A sharp form of an inequality of N. Trudinger, Indiana U. Math. J. 20, 1077-1092 (1971).

[54] C. Nash and S. Sen, Topology and Geometry for Physicists, Academic, London and New York, 1983.

[55] W.-M. Ni, On the elliptic equation $\Delta u+K(x) \mathrm{e}^{2 u}=0$ and conformal metrics with prescribed Gaussian curvatures, Invent. Math. 66, 343-352 (1982).

[56] M. Noguchi, Yang-Mills-Higgs theory on a compact Riemann surface, J. Math. Phys. 28, 2343-2346 (1987).

[57] M. Nolasco and G. Tarantello, On a sharp Sobolev type inequality on two dimensional compact manifolds, Arch. Rat. Mech. Anal. 145, 161-195 (1998).

[58] M. Nolasco and G. Tarantello, Double vortex condensates in the Chern-Simons-Higgs theory, Calc. Var. \& PDE's 9, 31-91 (1999). 
[59] T.H. Parker and C.H. Taubes, On Witten's proof of the positive energy theorem, Commun. Math. Phys. 84, 223-238 (1982).

[60] R. Penrose, Naked singularities, Ann. New York Acad. Sci. 224, 125-134 (1973)

[61] R. Rajaraman, Solitons and Instantons, North Holland, Amsterdam, 1982.

[62] R. Schoen and S.-T. Yau, Complete manifolds with nonnegative scalar curvature and the positive action conjecture in general relativity, Proc. Nat. Acad. Sci. 75, 45-76 (1978).

[63] R. Schoen and S.-T. Yau, On the proof of the positive mass conjecture in general relativity, Commun. Math. Phys. 65, 45-76 (1979).

[64] R. Schoen and S.-T. Yau, Positivity of the total mass of a general space-time, Phys. Rev. Lett. 43, 1457-1459 (1979).

[65] R. Schoen and S.-T. Yau, Proof of the positive mass theorem II, Commun. Math. Phys. 79, 231-260 (1981)

[66] B. J. Schroers, Bogomol'nyi solitons in a gauged $O(3)$ sigma model, Phys. Lett. B 356, 291-296 (1995).

[67] B. J. Schroers, The spectrum of Bogomol'nyi solitons in gauged linear sigma models, Nucl. Phys. B 475, 440-468 (1996).

[68] L. M. Sibner, R. J. Sibner, and Y. Yang, Abelian gauge theory on Riemann surfaces and new topological invariants, Proc. Roy. Soc. London A 456, 593-613 (2000).

[69] J. Smoller, A. Wasserman, S. T. Yau, and J. B. McLeod, Smooth static solutions of the Einstein-Yang/Mills equations Bull. Amer. Math. Soc. (N.S.) 27 (1992) 239-242; Comm. Math. Phys. 143 (1991) 115-147.

[70] C. H. Taubes, Arbitrary $N$-vortex solutions to the first order Ginzburg-Landau equations, Commun. Math. Phys. 72 (1980) 277-292.

[71] N. Trudinger, On embedding into Orlitz spaces and some applications, J. Math. Phys. 17, 473-484 (1967).

[72] A. Vilenkin, Cosmic strings and domain walls, Phys. Rep. 121, 263-315 (1985).

[73] A. Vilenkin and E. P. S. Shellard, Cosmic Strings and Other Topological Defects, Cambridge University Press, 1994.

[74] S. Wang and Y. Yang, Abrikosov's vortices in the critical coupling, SIAM J. Math. Anal. 23 (1992) 1125-1140.

[75] Wikipedia, a free online encyclopedia.

[76] E. Witten, A new proof of the positive energy theorem, Commun. Math. Phys. 80, 381-402 (1981).

[77] E. Witten, Superconducting strings, Nucl. Phys. B 249, 557-592 (1985).

[78] Y. Yang, Existence, regularity, and asymptotic behavior of the Ginzburg-Landau equations on $\mathbb{R}^{3}$, Commun. Math. Phys. 123, 147-161 (1989).

[79] Y. Yang, The existence of Ginzburg-Landau solution on the plane by a direct variational method, Ann. Inst. H. Poincaré - Anal. non linéaire 11, 517-536 (1994).

[80] Y. Yang, Obstructions to the existence of static cosmic strings in an Abelian Higgs model, Phys. Rev. Lett. 73, 10-13 (1994).

[81] Y. Yang, Prescribing topological defects for the coupled Einstein and Abelian Higgs equations, Commun. Math. Phys. 170 (1995) 541-582. 
[82] Y. Yang, Static cosmic strings on $S^{2}$ and criticality, Proc. Roy. Soc. London A 453 (1997) 581-591.

[83] Y. Yang, Self duality of the gauge field equations and the cosmological constant, Commun. Math. Phys. 162, 481-498 (1994).

[84] Y. Yang, Coexistence of vortices and antivortices in an Abelian gauge theory, Phys. Rev. Lett. 80, 26-29 (1998).

[85] Y. Yang, Strings of opposite magnetic charges in a gauge field theory, Proc. Roy. Soc. London A 455, 601-629 (1999).

[86] Y. Yang, Classical solutions in the Born-Infeld theory, Proc. Roy. Soc. London A 456, 615-640 (2000).

[87] Y. Yang, Solitons in Field Theory and Nonlinear Analysis, Springer-Verlag, New York, 2001.

[88] Y. Yang, Prescribing zeros and poles on a compact Riemann surface for a gravitationally coupled Abelian gauge field theory, Commun. Math. Phys. 249, 579-609 (2004).

Yisong Yang

Department of Mathematics

Polytechnic University

Brooklyn, New York 11201.

E-mail: yyang@math.poly.edu 